\title{
Wide vector solitons in systems with time- and space-modulated nonlinearities
}

\author{
L. E. Arroyo Meza, ${ }^{*}$ A. de Souza Dutra, ${ }^{\dagger}$ and M. B. Hott ${ }^{\ddagger}$ \\ UNESP Universidade Estadual Paulista, Campus de Guaratinguetá, DFQ, Avenida Dr. Ariberto Pereira da Cunha, \\ 333, 12516-410, Guaratinguetá, São Paulo, Brazil
}

(Received 14 June 2013; published 8 November 2013)

\begin{abstract}
In this work we apply point canonical transformations to solve some classes of two coupled nonautonomous nonlinear Schrödinger equations with specific cubic and quintic-time- and space-dependent—nonlinearities. The method applied here allows us to find a class of wide localized (in space) vector soliton solutions of nonautonomous nonlinear Schrödinger equations. The vector solitons found here can be applied to theoretical studies of Bose-condensed atoms in two different internal states and of ultrashort pulse propagation in optical fibers with focusing and defocusing nonlinearities.
\end{abstract}

DOI: 10.1103/PhysRevE.88.053202

PACS number(s): 05.45.Yv, 03.75.Lm, 42.65.Tg, 42.81.Dp

\section{INTRODUCTION}

Nonlinear physical phenomena have been objects of extensive theoretical and experimental studies for quite a long time. In particular, the ultrashort pulse propagation in optical fibers and waveguides as well as the dynamics of a Bose-Einstein condensate (BEC) are only a few examples of such nonlinear physical systems which find potential applications in different fields of science and technology. An optical fiber waveguide that can trap high-intensity electromagnetic fields (ultrashort pulse) inside a small core area presents a natural nonlinear medium where the transverse field distribution does not change on propagation, such that the field evolution problem is essentially one dimensional, making it very attractive for mathematical modeling [1]. In the case of BECs, the most remarkable characteristic of the system, its intrinsic nonlinearity, is due to the interatomic interactions among atoms cooled to temperatures very near absolute zero. In particular, the successful trapping of a BEC in a hollow blue-detuned laser beam [2] shows that a quasi-one-dimensional BEC can be experimentally realized.

The nonlinear Schrödinger equation (NLSE) is useful for describing the dynamics of such nonlinear one-dimensional phenomena as mentioned above. Specifically, the NLSE with cubic and quintic nonlinearities (CQNLSE) models a BEC when interactions between two atoms (cubic nonlinearity) and three atoms (quintic nonlinearity) are considered. Furthermore, the CNLSE describes the dynamics of a pulse propagating in a Kerr-nonlinearity medium, whereas the CQNLSE describes the dynamics of an ultrashort pulse propagating in a non-Kerr-nonlinearity medium.

There are a vast variety of methods to obtain exact analytical solutions of the NLSE in $1+1$ dimensions, such as the inverse scattering transform [3], the Hirota method [4], the similarity transformation method, which was applied for the first time to solve the nonautonomous CQSNLE in [5] and later in [6,7], and the point canonical transformations [8], which explains the origin of the ansatz involved in the similarity transformation method. The best-known solutions of the NLSE are those

\footnotetext{
*luisarroyo@feg.unesp.br

†dutra@feg.unesp.br

${ }^{\ddagger}$ marcelo.hott@pq.cnpq.br
}

for solitary waves or solitons [9]. Classical solitons appear in nonlinear and dispersive systems that are described by a NLSE with constant distributed coefficients (autonomous systems). Frequently, these systems have no significant effect on the manipulation of the soliton's shape. A more realistic situation is one in which a nonlinear and dispersive system is influenced by some external time- and/or space-dependent force (nonautonomous system) [10]. In this case, a NLSE with variable coefficients modulated in space and/or time describes the dynamics of the system. Modulation of these coefficients allows us to control the width, amplitude, and center of mass, while maintaining the overall shapes of the solutions, which are called nonautonomous solitons. For example, in a BEC it is possible to modulate the magnitude and sign of the two-body scattering length $a_{s}$, through the Feshbach resonance in an external magnetic [11] or optical field [12]. By means of this method and for the average intensity of the two-body interaction closes to zero, one can approach the Efimov limit, dominated by three-body interactions [13]. On the other hand, for the description of ultrashort pulse propagation in optical fibers it is necessary to take into account higher-order nonlinearities. Those arise from the expansion of the refractive index $n$ in powers of ultrashort pulse intensity $I$ : $n=n_{0}+n_{2} I+n_{4} I^{2}+\cdots$, where $n_{0}$ is the linear refractive index coefficient, $n_{2}=3 \chi^{(3)} / 8 n_{0}$ and $n_{4}=5 \chi^{(5)} / 32 n_{0}$ are the cubic and the quintic nonlinearity coefficients, respectively; $\chi^{(i)}$ is the $i$ th-order susceptibility. If the expansion is truncated at second-order terms with respect to $I$, the corresponding differential equation governing the nonlinear evolution of the ultrashort pulse in the optical fiber is the CQNLSE [14].

Specifically, bright and dark solitons have been observed in both nonlinear physical phenomena mentioned above. Investigation of bright and dark solitons is useful for understanding the properties of BECs and of ultrashort pulse propagation. Bright solitons are characterized by a localized maximum in the density profile without any phase jump across it. In the relevant experiments, this type of soliton is formed by utilizing a Feshbach resonance to change the sign of the scattering length from positive to negative. On the other hand, dark solitons may also be considered as moving domain walls which separate regions of a condensate with different values of the order parameter. In fact, dark solitons are density dips characterized by a phase jump of the wave function at the position of the dip and can be generated by means 
of phase-engineering techniques [15]. Thus, there are many possibilities for managing these solitons, strongly justifying a quest for further analytical solutions.

In addition to the bright and dark solitons, there are also various multicomponent (vector) solitons, which arise as solutions of systems of coupled NLSEs, which are used to describe the interaction among the components. For example, in the context of cold atomic gases, two vector components, whose dynamics are governed by the Gross-Pitaevskii equation or the NLSE, are the macroscopic wave functions of Bose-condensed atoms in two different hyperfine states $|1\rangle$ and $|2\rangle$.

In the context of nonlinear optical fibers, the two vector components correspond to the two orthogonal polarization axes, with two different frequencies, of the ultrashort pulse propagating in the fiber. This can be explained in the following way. When both polarization modes are excited in an optical fiber, the soliton polarization components propagate with different velocities, due to birefringence. At the same time, if the birefringence is moderate, the cross-phase modulation (XPM), which refers to the nonlinear phase change of an optical field induced by other copropagating fields, prevents soliton splitting. Formally, the problem may be related to that of soliton formation in a fiber without birefringence when the polarization components of the initial pulse have different frequencies. These facts explain the interest in the main features of the vector NLSE solitons investigated in the frameworks of both autonomous and nonautonomous NLSE models.

Vector solitons in the form of periodic-periodic, dark-dark, bright-bright, and dark-bright solitons obtained from a system of two coupled CNLSEs have been extensively studied [16].

In this work, we will find vector soliton solutions from a system of two coupled nonautonomous CQNLSEs by considering both two- and three-body interactions and nonKerr-nonlinearity media for the case of BECs and optical fibers, respectively.

Recently, in the work [17] attention is focused on two coupled nonautonomous NLSEs with cubic nonlinearity. The authors obtain analytical vector solitons of the following types: bright-bright, dark-dark, and bright-dark. Motivated by this work, we extend the problem, focusing our attention on two coupled nonautonomous NLSEs in one space dimension with cubic and quintic nonlinearities and amplification or absorption coefficients. To search for different vector solitons of two coupled nonautonomous CQNLSEs, we employ the following strategy. First, point canonical transformations [8] are used to transform the two coupled nonautonomous CQNLSEs into two coupled autonomous CQNLSEs. Next, we make an analogy between those equations and the static equations of motion of a model of two interacting scalar fields in supersymmetric field theory in $1+1$ dimensions [18]. We work with topological solutions of the latter model, because they are classically stable. Finally, this analogy allows us to use the orbit equation method [19] to find topological solutions of the two coupled autonomous CQNLSEs. With this method an integration constant shows up naturally, which controls the width and the amplitude of the topological solution when the constant is close to its critical value [20]. As we show, this strategy leads to several solutions which can be continuously deformed into wide vector solitons by tuning the integration constant. At the critical value of the integration constant a gray-dark soliton turns into a dark-dark soliton. Those behaviors are illustrated in a series of examples which might be useful in describing BECs and/or ultrashort pulse propagation in optical fibers.

\section{THEORETICAL MODEL}

In order to describe the dynamics of ultrashort pulse propagation along orthogonal polarization axes in nonlinear optical fibers with Kerr and non-Kerr nonlinearities we consider the following system of coupled nonautonomous NLSEs with terms of cubic and quintic order in the wave function:

$$
\begin{aligned}
i \frac{\partial \Psi_{1}}{\partial t}= & -m_{1}(t) \frac{\partial^{2} \Psi_{1}}{\partial x^{2}}+v_{1}(x, t) \Psi_{1} \\
& +\left(\sum_{k=1}^{2} g_{1 k}(x, t)\left|\Psi_{k}\right|^{2}\right) \Psi_{1}+\left(\sum_{k=1}^{2} f_{1 k}(x, t)\left|\Psi_{k}\right|^{4}\right) \Psi_{1} \\
& +h_{12}(x, t)\left|\Psi_{1}\right|^{2}\left|\Psi_{2}\right|^{2} \Psi_{1}+i \Gamma_{1}(t) \Psi_{1}, \\
i \frac{\partial \Psi_{2}}{\partial t}= & -m_{2}(t) \frac{\partial^{2} \Psi_{2}}{\partial x^{2}}+v_{2}(x, t) \Psi_{2} \\
& +\left(\sum_{k=1}^{2} g_{2 k}(x, t)\left|\Psi_{k}\right|^{2}\right) \Psi_{2}+\left(\sum_{k=1}^{2} f_{2 k}(x, t)\left|\Psi_{k}\right|^{4}\right) \Psi_{2} \\
& +h_{21}(x, t)\left|\Psi_{1}\right|^{2}\left|\Psi_{2}\right|^{2} \Psi_{2}+i \Gamma_{2}(t) \Psi_{2},
\end{aligned}
$$

where $\Psi_{k}=\Psi_{k}(x, t)$ and $v_{k}(x, t)$ are the complex envelope of the electric field in a comoving frame and the trapping potential, respectively. The functions $g_{k l}(x, t), f_{k l}(x, t)$, and $h_{k l}(x, t)$ (from now on we consider $k, l=1,2$ ) describe the strength management of the cubic and quintic (Kerr and non-Kerr) nonlinearities, respectively, $m_{k}(t)$ is the dispersion parameter, and $\Gamma_{k}(t)$ is the amplification $(\Gamma>0)$ or the absorption $(\Gamma<0)$ coefficient. The continuity equation of the system is of the form $\frac{\partial \rho}{\partial t}+\frac{\partial J}{\partial x}=2 \sum_{k=1}^{2} \Gamma_{k}(t)\left|\Psi_{k}\right|^{2}$, where $\rho=\sum_{k=1}^{2}\left|\Psi_{k}\right|^{2}$ and $J=\sum_{k=1}^{2}-i m_{k}(t)\left(\Psi_{k}^{*} \frac{\partial \Psi_{k}}{\partial x}-\Psi_{k} \frac{\partial \Psi_{k}^{*}}{\partial x}\right)$, that is, the system is conservative only when $\Gamma_{k}(t)=0$.

In the case of $m_{1}(t)=m_{2}(t)= \pm \frac{1}{2}, g_{11}(x, t)=g_{22}(x, t)=$ $-1, g_{12}(x, t)=g_{21}(x, t)=-\kappa$ and the remaining coefficients zero, Eqs. (1) and (2) reduce to the integrable Manakov system [21], where $\kappa$ is the XPM coefficient, which is equal to 1 in the Manakov model and to 2/3 in a linear birefringent optical fiber. Nontrivial interaction scenarios of Manakov's solitons were discovered and analyzed for the first time in [22]. In that case, however, the authors analyze an autonomous system; nevertheless it would be very interesting to see what happens with the interaction in the case of a nonautonomous system like the ones which are considered here. In fact, we have been able to implement generalizations of [22-24] by taking into account time-dependent nonlinearities. The results are under analysis and we intend to report them elsewhere.

\section{THE APPROACH}

We shall proceed in the same manner as in [8]. In this case, we consider that $m_{k}(t), v_{k}(x, t), g_{k l}(x, t), f_{k l}(x, t), h_{k l}(x, t)$, and 
$\Gamma_{k}(t)$ have the forms

$$
v_{k}(x, t)=\omega(t) x^{2}+f_{1}(t) x+f_{2}(t)+T(t) V_{k}[\gamma(t) x+\delta(t)],
$$

$$
\begin{aligned}
& g_{k l}(x, t)=G_{k l} T(t) \beta_{l}(t) g[\gamma(t) x+\delta(t)], \\
& f_{k l}(x, t)=F_{k l} T(t) \beta_{l}^{2}(t) f[\gamma(t) x+\delta(t)],
\end{aligned}
$$

$$
\begin{aligned}
h_{k l}(x, t) & =H_{k l} T(t) \beta_{k}(t) \beta_{l}(t) h[\gamma(t) x+\delta(t)] \quad \text { only for } \\
k & \neq l, \\
\Gamma_{k}(t) & =-\frac{1}{2} \frac{d}{d t}\left\{\ln \left[\beta_{k}(t) \gamma(t)\right]\right\}, \quad m_{k}(t)=\frac{T(t)}{\gamma^{2}(t)} .
\end{aligned}
$$

The two coupled nonautonomous CQNLSEs (1) and (2) are written in standard soliton units, as they are commonly known. If we choose $T(t)=\gamma^{2}(t), \beta_{k}(t)=\gamma^{-1}(t)$, i.e., $m_{k}(t)=$ 1 (constant mass) and $\Gamma_{k}(t)=0$ (conservative system), and $F_{k l}=H_{k l}=0$ the two coupled nonautonomous CQNLSEs will model a system of two-component BECs, which are composed of two different hyperfine atomic levels of the same atom.

Now, we implement the following coordinate transformation and time rescaling:

$$
x=\frac{\xi}{\bar{\gamma}(\tau)}-\frac{\bar{\delta}(\tau)}{\bar{\gamma}(\tau)}, \quad t-t_{0}=\int_{0}^{\tau} \frac{d \tau^{\prime}}{\bar{T}\left(\tau^{\prime}\right)}
$$

with $\bar{\gamma}[\tau(t)]=\gamma(t), \quad \bar{\delta}[\tau(t)]=\delta(t)$, and $\bar{T}[\tau(t)]=T(t)$. Then, Eqs. (1) and (2) can be written as

$$
\begin{aligned}
& i \frac{\bar{T}}{\bar{\gamma}}\left(\bar{\gamma}_{\tau} \xi-\bar{\gamma}_{\tau} \bar{\delta}+\bar{\gamma}_{\tau}\right) \frac{\partial \bar{\Psi}_{i}}{\partial \xi}+i \bar{T} \frac{\partial \bar{\Psi}_{i}}{\partial \tau} \\
& =-\bar{m}_{i} \bar{\gamma}^{2} \frac{\partial^{2} \bar{\Psi}_{i}}{\partial \xi^{2}}+\bar{v}_{i}(\xi, \tau) \bar{\Psi}_{i} \\
& \quad+\left(\sum_{k=1}^{2} G_{i k} \bar{T} \bar{\beta}_{k} \bar{g}(\xi)\left|\bar{\Psi}_{k}\right|^{2}\right) \bar{\Psi}_{i} \\
& \quad+\left(\sum_{k=1}^{2} F_{i k} \bar{T} \bar{\beta}_{k}^{2} \bar{f}(\xi)\left|\bar{\Psi}_{k}\right|^{4}\right) \bar{\Psi}_{i} \\
& \quad+\left(\sum_{k=1}^{2} G_{i k} \bar{T} \bar{\beta}_{k} \bar{g}(\xi)\left|\bar{\Psi}_{k}\right|^{2}\right) \bar{\Psi}_{i} \\
& \quad+\left(\sum_{k=1}^{2} F_{i k} \bar{T} \bar{\beta}_{k}^{2} \bar{f}(\xi)\left|\bar{\Psi}_{k}\right|^{4}\right) \bar{\Psi}_{i} \\
& \quad+H_{i k} \bar{T}_{i} \bar{\beta}_{k} \bar{h}(\xi)\left|\bar{\Psi}_{i}\right|^{2}\left|\bar{\Psi}_{k}\right|^{2} \bar{\Psi}_{i}+i \bar{\Gamma}_{i} \bar{\Psi}_{i},
\end{aligned}
$$

where the index $i=1,2$ stands for Eqs. (1) and (2), respectively, and $H_{i k} \bar{\beta}_{i} \bar{\beta}_{k} \neq 0$ only for $i \neq k$. Moreover, $\bar{\gamma}_{\tau}=$ $d \bar{\gamma} / d \tau, \bar{\delta}_{\tau}=d \bar{\delta} / d \tau, \bar{\Psi}_{i}=\Psi_{i}(x(\xi, \tau), t(\tau)), \bar{\Gamma}_{i}[\tau(t)]=\Gamma_{i}(t)$, and

$$
\begin{aligned}
\bar{v}_{i}(\xi, \tau)= & \bar{\omega}(\tau) \frac{(\xi-\bar{\delta})^{2}}{\bar{\gamma}^{2}}+\bar{f}_{1}(\tau) \frac{(\xi-\bar{\delta})}{\bar{\gamma}}+\bar{f}_{2}(\tau) \\
& +\bar{T}(\tau) \bar{V}_{i}(\xi) .
\end{aligned}
$$

From the last three equations one can appreciate why we have chosen the specific dependence of $V_{k}, g, f$, and $h$ on $\xi=$ $\gamma(t) x+\delta(t)$ and the specific dependence of $m_{k}$ on $\gamma(t)$ and $T(t)$. The term with a first derivative in the variable $\xi$ in (9) can be eliminated by means of the redefinition

$$
\bar{\Psi}_{i}(\xi, \tau)=\frac{e^{-i \bar{\alpha}(\xi, \tau)}}{\sqrt{\bar{\beta}_{i}(\tau)}} \psi_{i}(\xi, \tau),
$$

where $\bar{\alpha}(\xi, \tau)=\frac{\bar{\gamma}_{\tau}}{4 \bar{\gamma}}(\xi-\bar{\delta})^{2}+\frac{\bar{\delta}_{\tau}}{2}(\xi-\bar{\delta})-\bar{a}(\tau)$, with $\bar{a}(\tau)$ an arbitrary function for the moment. The two functions $\psi_{i}(\xi, \tau)$ satisfy the set of two coupled time-dependent NLSEs compactly written as

$$
\begin{aligned}
i \frac{\partial \psi_{i}}{\partial \tau}= & -\frac{\partial^{2} \psi_{i}}{\partial \xi^{2}}+U_{i}(\xi, \tau) \psi_{i}+\left(\sum_{k=1}^{2} G_{i k} \bar{g}(\xi)\left|\psi_{k}\right|^{2}\right) \psi_{i} \\
& +\left(\sum_{k=1}^{2} F_{i k} \bar{f}(\xi)\left|\psi_{k}\right|^{4}\right) \psi_{i}+H_{i k} \bar{h}(\xi)\left|\psi_{i}\right|^{2}\left|\psi_{k}\right|^{2} \psi_{i} \\
& +i\left(\frac{\bar{\Gamma}_{i}}{\bar{T}}+\frac{\partial^{2} \bar{\alpha}}{\partial \xi^{2}}+\frac{d}{d \tau} \ln \bar{\beta}_{i}^{1 / 2}\right) \psi_{i}
\end{aligned}
$$

where $U_{i}(\xi, \tau)=\left(\frac{\bar{\omega}(\tau)}{\bar{T}}-\frac{\bar{\gamma}_{\tau \tau} \bar{\gamma}}{4}\right) \frac{(\xi-\bar{\delta})^{2}}{\bar{\gamma}^{2}}+\left(\frac{\bar{f}_{1}(\tau)}{\bar{T}}-\frac{\bar{\delta}_{\tau \tau} \bar{\gamma}}{2}\right) \frac{(\xi-\bar{\delta})}{\bar{\gamma}}+$ $\left(\frac{\bar{f}_{2}(\tau)}{\bar{T}}+\frac{\bar{\delta}_{\tau}^{2}}{4}+\frac{d \bar{a}}{d \tau}\right)+V_{i}(\xi)$. One can see why the factors involving $T(t)$ are present in the expressions of $v_{k}(x, t), g_{k l}(x, t)$, $f_{k l}(x, t)$, and $h_{k l}(x, t)$. Moreover, one can also appreciate the contribution of the redefinition (11), specifically the contribution of the phase $\bar{\alpha}(\xi, \tau)$ to the redefinition of the trapping potential $U_{k}(\xi, \tau)$ and the dependence of $\Gamma_{k}$ on $\gamma(t)$, $\beta_{k}(t)$, and their first derivatives.

Now, we choose

$$
\begin{aligned}
\bar{\omega}(\tau) & =\frac{\bar{T} \bar{\gamma}_{\tau \tau} \bar{\gamma}}{4}, \quad \bar{f}_{1}(\tau)=\frac{\bar{T} \bar{\delta}_{\tau \tau} \bar{\gamma}}{2}, \\
\bar{f}_{2}(\tau) & =-\bar{T}\left(\frac{\bar{\delta}_{\tau}^{2}}{4}+\frac{d \bar{a}}{d \tau}\right),
\end{aligned}
$$

such that in terms of $t$ one has

$$
\begin{aligned}
\omega(t) & =\frac{\gamma}{4} \frac{d}{d t}\left(\frac{\gamma_{t}}{T}\right), \quad f_{1}(t)=\frac{\gamma}{2} \frac{d}{d t}\left(\frac{\delta_{t}}{T}\right), \\
f_{2}(t) & =-\frac{\delta_{t}^{2}}{4 T}-\frac{d a}{d t},
\end{aligned}
$$

which reveal the intrinsic connection between the frequency $\omega(t)$, the force $f_{1}(t)$, and the functions $\gamma(t), \delta(t)$, and $T(t)$. Note that $a(t)$ is an arbitrary function that could be chosen as $d a / d t=\delta_{t}^{2} / 2 T$ if $f_{2}(t)$ was not present in (3). Thus, one removes the explicit dependence of (12) on $\tau$, that is,

$$
\begin{aligned}
i \frac{\partial \psi_{i}}{\partial \tau}= & -\frac{\partial^{2} \psi_{i}}{\partial \xi^{2}}+V_{i}(\xi) \psi_{i}+\left(\sum_{k=1}^{2} G_{i k} \bar{g}(\xi)\left|\psi_{k}\right|^{2}\right) \psi_{i} \\
& +\left(\sum_{k=1}^{2} F_{i k} \bar{f}(\xi)\left|\psi_{k}\right|^{4}\right) \psi_{i}+H_{i k} \bar{h}(\xi)\left|\psi_{i}\right|^{2}\left|\psi_{k}\right|^{2} \psi_{i}
\end{aligned}
$$


We notice that the wave functions (11) are written in the original variables $(x, t)$ as

$$
\Psi_{k}(x, t)=\frac{e^{-i \alpha(x, t)}}{\sqrt{\beta_{k}(t)}} \psi_{k}(\xi(x, t), \tau(t)),
$$

where $\alpha(x, t)=\frac{\gamma_{t} \gamma}{4 T} x^{2}+\frac{\delta_{t} \gamma}{2 T} x-a(t)$, and recall that the phase $\alpha(x, t)$ was introduced through the redefinition (11) and whose eventual contribution to the redefinition of the trapping potential can be eliminated thanks to the presence of the time-dependent driven harmonic oscillator terms [the three first terms of $v(x, t)]$.

By looking for stationary solutions in the variable $\tau$, we set $\psi_{i}(\xi, \tau)=\phi_{i}(\xi) \exp \left(-i E_{i} \tau\right)$, such that $\phi_{i}(\xi)$ satisfies

$$
\begin{aligned}
\frac{d^{2} \phi_{i}}{d \xi^{2}}= & {\left[V_{i}(\xi)-E_{i}\right] \phi_{i}+\left(\sum_{k=1}^{2} G_{i k} \bar{g}(\xi)\left|\phi_{k}\right|^{2}\right) \phi_{i} } \\
& +\left(\sum_{k=1}^{2} F_{i k} \bar{f}(\xi)\left|\phi_{k}\right|^{4}\right) \phi_{i}+H_{i k} \bar{h}(\xi)\left|\phi_{i}\right|^{2}\left|\phi_{k}\right|^{2} \phi_{i}
\end{aligned}
$$

Since we still have two coupled nonlinear differential equations with inhomogeneous nonlinearities, we are going to make further transformations in order to reach two coupled nonlinear second-order differential equations with homogeneous coefficients. For that we impose the requirement that $\xi$ is a function of another variable $\zeta$, that is, $\xi=\bar{\Theta}(\zeta)$, and redefine $\phi_{i}$ in terms of fields $\Phi_{i}(\zeta)$ as

$$
\bar{\phi}_{i}(\zeta)=\sqrt{\bar{\Theta}_{\zeta}} \Phi_{i}(\zeta)
$$

which obey the following equations:

$$
\begin{aligned}
\frac{d^{2} \Phi_{i}}{d \zeta^{2}}= & -\mu_{i} \Phi_{i}+\left(\sum_{k=1}^{2} G_{i k}\left|\Phi_{k}\right|^{2}\right) \Phi_{i}+\left(\sum_{k=1}^{2} F_{i k}\left|\Phi_{k}\right|^{4}\right) \Phi_{i} \\
& +H_{i k}\left|\Phi_{i}\right|^{2}\left|\Phi_{k}\right|^{2} \Phi_{i},
\end{aligned}
$$

where

$$
\begin{gathered}
\mu_{i}=-\bar{\Theta}_{\zeta}^{2}\left\{V_{i}[\xi(\zeta)]-E_{i}\right\}+\frac{\bar{\Theta}_{\zeta \zeta \zeta}}{2 \bar{\Theta}_{\zeta}}-\frac{3 \bar{\Theta}_{\zeta \zeta}^{2}}{4 \bar{\Theta}_{\zeta}^{2}}, \\
\bar{g}[\xi(\zeta)]=\frac{1}{\bar{\Theta}_{\zeta}^{3}}, \quad \bar{f}[\xi(\zeta)]=\bar{h}[\xi(\zeta)]=\frac{1}{\bar{\Theta}_{\zeta}^{4}} .
\end{gathered}
$$

As one can see, we have not defined the parts $V_{i}[\xi(\zeta)]$ of the trapping potentials $v_{i}$ yet. As a matter of fact, $V_{1}(\xi)$ will differ slightly from $V_{2}(\xi)$, once they are defined in terms of $\Theta(\xi)$ and the constant chemical potentials $\mu_{i}$. In terms of the variables $\xi$ we have

$$
\begin{aligned}
V_{i}(\xi) & =\left(\frac{\Theta^{\prime \prime}}{2 \Theta^{\prime}}\right)^{2}-\frac{d}{d \xi}\left(\frac{\Theta^{\prime \prime}}{2 \Theta^{\prime}}\right)-\mu_{i} \Theta^{\prime 2}+E_{i}, \\
\bar{g}(\xi) & =\Theta^{\prime 3}, \quad \bar{f}(\xi)=\bar{h}(\xi)=\Theta^{\prime 4},
\end{aligned}
$$

where $\Theta^{\prime}=d \Theta / d \xi$ (parenthetically we notice that when $\mu_{i}=E_{i}=0$ then $V_{1}=V_{2}$, which manifests itself as a supersymmetric quantum mechanics potential on the variable $\xi)$, and the eigenfunctions (18) become

$$
\phi_{i}(\xi)=\frac{1}{\sqrt{\Theta^{\prime}(\xi)}} \Phi_{i}[\zeta(\xi)] \quad(i=1,2) .
$$

Finally, by returning to the original space-time coordinates $(x, t)$, the wave functions are given by

$$
\begin{aligned}
\Psi_{i}(x, t) & =\frac{1}{\sqrt{\beta_{i}(t) \Theta^{\prime}[\xi(x, t)]}} \exp \left[-i \eta_{i}(x, t)\right] \Phi_{i}\{\zeta[\xi(x, t)]\} \\
(i & =1,2)
\end{aligned}
$$

with $\eta_{i}(x, t)=\frac{\gamma_{t} \gamma}{4 T} x^{2}+\frac{\delta_{t} \gamma}{2 T} x-a(t)+E_{i} \int_{0}^{t} d t^{\prime} T\left(t^{\prime}\right)$, where $a(t)$ is an arbitrary function.

Thus, we have shown by means of point canonical transformations how two specific coupled nonautonomous CQNLSEs, Eqs. (1) and (2), can be mapped into two coupled autonomous nonlinear second-order differential equations which are represented compactly in (19). Moreover, in order to reach (19) the parts of the trapping potentials $V_{k}(\xi)$ and the nonlinearity functions $g(\xi), f(\xi)$, and $h(\xi)$ must be related to each other by means of only one function $\Theta(\xi)=\bar{\Theta}^{-1}(\zeta(\xi))$ [see Eqs. (21)].

\section{EXACT SOLUTIONS}

To treat the two coupled autonomous CQNLSEs in (19) we first suppose that those equations are the static equations of motion, with $\zeta$ playing the role of space coordinate, for the bosonic sector of a relativistic supersymmetric model in $1+1$ space-time dimensions. In such a presumed supersymmetric model, $\Phi_{1}$ and $\Phi_{2}$ are two interacting real scalar fields. As it is well known [18], the minimum-energy solutions found for this bosonic sector of a supersymmetric model are topological solitons of the Bogomol'nyi-Prasad-Sommerfield (BPS) and non-BPS types. Then $\Phi_{1}$ and $\Phi_{2}$ are classical static configurations on $\zeta$ and Eqs. (19) are written as

$$
\frac{d^{2} \Phi_{1}}{d \zeta^{2}}=\frac{\partial U\left(\Phi_{1}, \Phi_{2}\right)}{\partial \Phi_{1}}, \quad \frac{d^{2} \Phi_{2}}{d \zeta^{2}}=\frac{\partial U\left(\Phi_{1}, \Phi_{2}\right)}{\partial \Phi_{2}},
$$

where $U\left(\Phi_{1}, \Phi_{2}\right)$ is the interaction potential of the model. Thus, due to the equality $\frac{\partial^{2} U\left(\Phi_{1}, \Phi_{2}\right)}{\partial \Phi_{2} \partial \Phi_{1}}=\frac{\partial^{2} U\left(\Phi_{1}, \Phi_{2}\right)}{\partial \Phi_{1} \partial \Phi_{2}}$, we find that the coefficients of the nonlinear terms in (19) must be related to each other by $G_{12}=G_{21}, H_{12}=2 F_{21}$, and $H_{21}=2 F_{12}$. For the bosonic sector of a supersymmetric model one can write

$$
U\left(\Phi_{1}, \Phi_{2}\right)=\frac{1}{2}\left[\left(\frac{\partial W}{\partial \Phi_{1}}\right)^{2}+\left(\frac{\partial W}{\partial \Phi_{2}}\right)^{2}\right],
$$

where $W\left(\Phi_{1}, \Phi_{2}\right)$ is the so-called superpotential. We will work with BPS solutions, because they are classically stable at least at the level of the classical field model in $1+1$ space-time dimensions [25]. In this case it can be shown that the solutions of the BPS equations

$$
\frac{d \Phi_{1}}{d \zeta}=\frac{\partial W\left(\Phi_{1}, \Phi_{2}\right)}{\partial \Phi_{1}}, \quad \frac{d \Phi_{2}}{d \zeta}=\frac{\partial W\left(\Phi_{1}, \Phi_{2}\right)}{\partial \Phi_{2}},
$$

are also solutions of the static equations of motion (24) and are those that saturate the lower-bound energy of the system, also known as the BPS energy. It is important to remark that the stability of the solutions at the level of the classical field model in $1+1$ space-time dimensions does not guarantee the stability 
of the solutions in (23) for the original nonautonomous NLSEs (1) and (2).

In order to find solutions of (26) we use the method developed in [19]. Summarily, this method consists in integrating the differential equation $\frac{d \Phi_{1}}{d \Phi_{2}}=\frac{\partial W / \partial \Phi_{1}}{\partial W / \partial \Phi_{2}}$, leading us to an orbit equation which relates the solutions for both fields that minimize the energy of the system. Once a constant of integration (hereafter $c_{0}$ ) comes up naturally from the integration, one finds a variety of BPS solutions by varying $c_{0}$. One can find, for example, two-kink solutions for one of the fields and wide lump solutions for the other field, such that they can be continuously deformed by varying $c_{0}$. We refer the reader to Refs. [19], [20], and [26] to appreciate the details of the role played by $c_{0}$ in the variety of solutions and the consequences of such solutions in specific models.

Here, the variety of BPS solutions implies a variety of wave functions $\Psi_{k}(x, t)$ and, consequently, many vector solitons for which real world applications might be found.

\section{EXAMPLES}

In this section, we show by means of some examples that the width and the amplitude of the solitons can be manipulated theoretically by focusing on some specific forms of the functions $\Theta(\xi), \gamma(t), \delta(t), \beta_{k}(t)$, and $T(t)$ but with some different values of $\mu_{k}, G_{k l}, F_{k l}$, and $H_{k l}$. We are going to consider two different cases; one of them is related to the modeling of BECs with two internal states and the other one models pulse propagation in a nonlinear optical fiber.

\section{A. BEC with two internal states (case A)}

When the two coupled nonautonomous CNLSEs model BECs with two different internal states, we consider $T(t)=$ $\gamma^{2}(t)$ and $\beta_{i}(t)=\gamma^{-1}(t)$. With this choice we have $m_{i}(t)=$ 1 (constant mass) and $\Gamma_{i}(t)=0$ (conservative system). We also choose $E_{1}=E_{2}=E>1, \mu_{1}=\mu_{2}=\mu$, and

$$
\begin{aligned}
& \Theta(\xi)=\int \sqrt{\frac{(E-1-q)(E-q)(E-1)}{\mu\left[E-1-q+q \operatorname{sn}^{2}(\xi \mid q)\right]^{2}}} d \xi, \\
& \gamma(t)=\frac{2 \gamma_{0}}{5-2 \sin (2 v t)}, \quad \delta(t)=0, \quad a(t)=0,
\end{aligned}
$$

such that the trapping potential for both components is composed of a harmonic and a periodic potential,

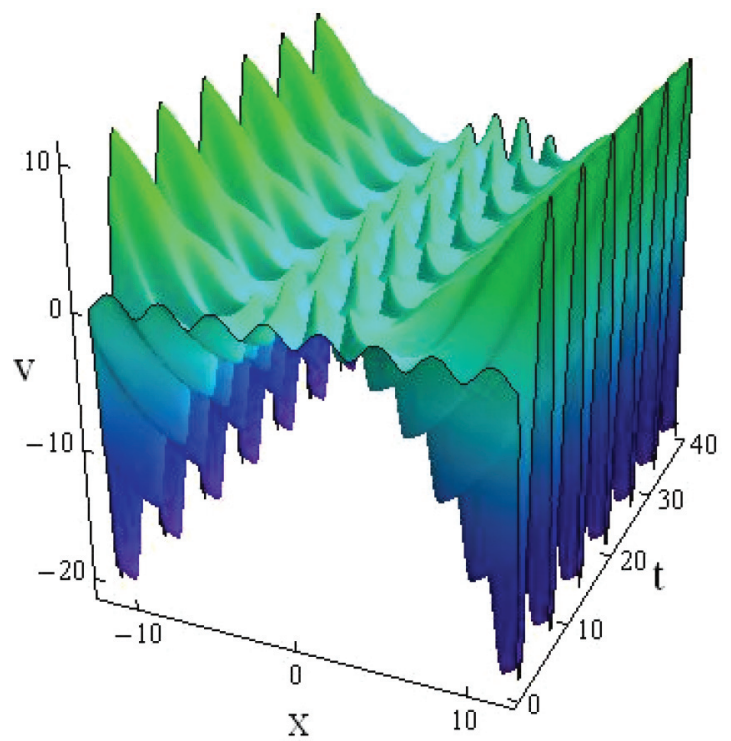

FIG. 1. (Color online) Trapping potential for BECs, Eq. (28), with $q=0.5, v=0.5$, and $\gamma_{0}=3$.

namely,

$$
v_{i}(x, t)=\omega(t) x^{2}+2 q \gamma^{2}(t) \operatorname{sn}^{2}(\xi \mid q),
$$

i.e., a magnetic trap and an optical lattice, which are important ingredients of experimental BEC setups [27] (Figure 1 shows this trapping potential.) In the above expression, $\operatorname{sn}(\xi \mid q)$ is the Jacobi elliptic function defined by $\operatorname{sn}(\xi \mid q)=\sin \theta$, with respect to the integral $\xi=\int_{0}^{\theta} d \alpha /\left(1-q \sin ^{2} \alpha\right)^{1 / 2}$, where $\theta$ is called the amplitude and $q(0 \leqslant q \leqslant 1)$ is the elliptic parameter [28].

Moreover, the choice of $\Theta(\xi)$ implies that the nonlinearities $g_{k l}(x, t), f_{k l}(x, t)$, and $h_{k l}(x, t)$ are periodic functions in space and time, which is a desirable feature for the coefficients that describe the strength management of the interaction between two atoms $\left[g_{k l}(x, t)\right]$, and three atoms $\left[f_{k l}(x, t)\right.$ and $\left.h_{k l}(x, t)\right]$.

\section{B. Ultrashort pulse propagation (case B)}

In the case where the two coupled nonautonomous CQNLSEs model ultrashort pulse propagation along orthogonal polarization axes in nonlinear optical fibers, we consider $T(t)=\gamma_{t}(t), \delta(t)=0, a(t)=0, E_{k}=\mu_{k}$, and $\Theta(\xi)=\xi$; with this choice we have $v_{k}(x, t)=0$. Moreover, we choose

$$
\begin{aligned}
\gamma(t) & =\frac{\beta_{0} \gamma_{0}^{2}\left(\Omega^{2}+\sigma^{2}\right)}{\sigma-e^{\sigma t}[\sigma \cos (\Omega t)+\Omega \sin (\Omega t)]+\beta_{0} \gamma_{0}\left(\Omega^{2}+\sigma^{2}\right)}, \\
\beta_{k}(t) & =\frac{e^{-\sigma t}\left\{\sigma-e^{\sigma t}[\sigma \cos (\Omega t)+\Omega \sin (\Omega t)]+\beta_{0} \gamma_{0}\left(\Omega^{2}+\sigma^{2}\right)\right\}^{2}}{\beta_{0} \gamma_{0}^{2}\left(\Omega^{2}+\sigma^{2}\right)^{2}},
\end{aligned}
$$

where $\gamma_{0}=\gamma(0), \beta_{0}=\beta_{k}(0)$, and $\left|\beta_{0} \gamma_{0} \omega\right|>1$, such that the dispersion parameters $m_{k}(t)=e^{\sigma t} \cos (\Omega t) / \beta_{0} \gamma_{0}^{2}$ and the cubic Kerr nonlinearity $g_{k l}(x, t)=G_{k l} \cos (\Omega t)$ as in Refs. [5,29]. In this case the quintic non-Kerr nonlinearity $\frac{f_{k l}(x, t)}{F_{k l}}=$ $\frac{h_{k l}(x, t)}{H_{k l}}=\frac{e^{-\sigma t} \cos (\Omega t)\left\{\sigma-e^{\sigma t}[\sigma \cos (\Omega t)+\Omega \sin (\Omega t)]+\beta_{0} \gamma_{0}\left(\Omega^{2}+\sigma^{2}\right)\right\}^{2}}{\beta_{0} \gamma_{0}^{2}\left(\Omega^{2}+\sigma^{2}\right)^{2}} \quad$ and the amplification or absorption parameters $\Gamma_{k}(t)=$ $\frac{\sigma^{2}+e^{\sigma t} \Omega[\Omega \cos (\Omega t)-\sigma \sin (\Omega t)]+\beta_{0} \gamma_{0} \sigma\left(\Omega^{2}+\sigma^{2}\right)}{2\left\{\sigma-e^{\sigma t}[\sigma \cos (\Omega t)+\Omega \sin (\Omega t)]+\beta_{0} \gamma_{0}\left(\Omega^{2}+\sigma^{2}\right)\right\}}$ are periodic functions. When $\sigma=0$ there is a balance (cancellation) between the effects generated by the dispersion parameters $m_{k}(t)$, by the Kerr and non-Kerr nonlinearities (refraction effects) $g_{k l}(x, t), f_{k l}(x, t), h_{k l}(x, t)$, and those effects generated by the absorption or amplification coefficients $\Gamma_{k}(t)$. 

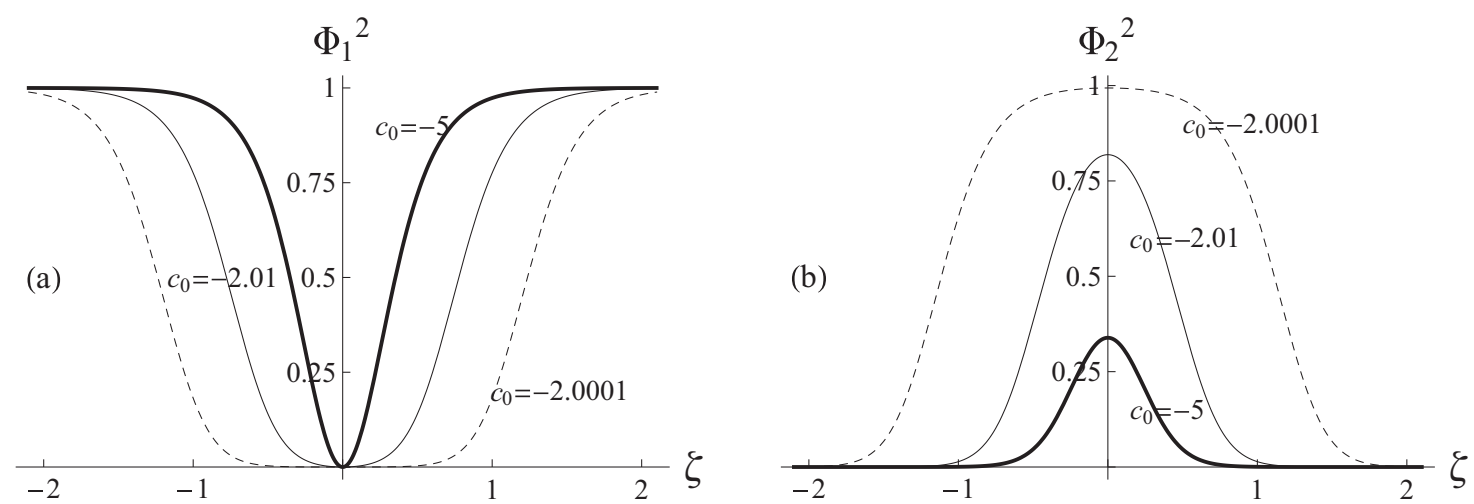

FIG. 2. Profiles of the components of the wide dark-bright vector soliton, Eq. (31). (a) Dark-type profile $\Phi_{1}^{2}(\zeta)$. (b) Bright-type profile $\Phi_{2}^{2}(\zeta) \cdot \lambda=2.5$.

\section{Example 1: Wide vector solitons under cubic nonlinearity}

In this example, the system of differential equations (1) and (2) can model Bose-condensed atoms in two different internal states or optical pulse propagation along orthogonal polarization axes in a nonlinear optical fiber with only Kerr nonlinearity (cubic nonlinearity). To find analytic solutions for Eqs. (19) we use the technique of the orbit equation [19]. To perform this technique, we choose $\mu_{k}=2 \lambda^{2}, G_{11}=G_{22}=2 \lambda^{2}, G_{12}=G_{21}=6 \lambda^{2}$, and $H_{k l}=$ $F_{k l}=0$. With this choice of parameters the second-order differential equations (19) are reduced to first-order differential equations:

$$
\frac{d \Phi_{1}}{d \zeta}=\lambda \Phi_{1}^{2}+\lambda \Phi_{2}^{2}-\lambda, \quad \frac{d \Phi_{2}}{d \zeta}=2 \lambda \Phi_{1} \Phi_{2}
$$

The orbit equation is

$$
\Phi_{1}^{2}=1+c_{0} \Phi_{2}+\Phi_{2}^{2}
$$

where $c_{0}$ is a constant of integration whose critical value is -2 [20].
The solutions of (30) are [19,26]

$$
\begin{aligned}
\Phi_{1}(\zeta) & =-\frac{\sqrt{c_{0}^{2}-4} \sinh (2 \lambda \zeta)}{\sqrt{c_{0}^{2}-4} \cosh (2 \lambda \zeta)-c_{0}} \\
\Phi_{2}(\zeta) & = \pm \frac{2}{\sqrt{c_{0}^{2}-4} \cosh (2 \lambda \zeta)-c_{0}} \text { for } c_{0}<-2 \\
\Phi_{1}(\zeta) & =-\frac{\tanh (\lambda \zeta) \mp 1}{2}, \quad \Phi_{2}(\zeta)=\frac{1 \pm \tanh (\lambda \zeta)}{2} \text { for } \\
c_{0} & =-2
\end{aligned}
$$

These solutions, for $c_{0}<-2$, exhibit dark-type and brighttype profiles, as shown in Fig. 2. When $c_{0}$ approaches its critical value, the dark-type soliton widens, while the amplitude and the width of the bright-type soliton increase.

In the case A of Bose-condensed atoms with two different internal states and with oscillatory repulsive interaction $\left[g_{k l}(x, t)>0\right.$ ],$\Psi_{1}(x, t)$ is a dark soliton and $\Psi_{2}(x, t)$ is a bright one, which can be found in single-component condensates only if they have negative scattering length [the atoms have an attractive interaction, $\left.g_{k l}(x, t)<0\right]$. The components of the vector wide dark-bright solitons are

$$
\begin{aligned}
\Psi_{1}(x, t)= & -\sqrt{\frac{2 \sqrt{2} \gamma_{0} \lambda\left[E-1-q+q \operatorname{sn}^{2}(\xi, q)\right]}{[5-2 \sin (2 v t)] \sqrt{(E-1-q)(E-q)(E-1)}}} \frac{\sinh [2 \lambda \Theta(\xi)]}{\cosh [2 \lambda \Theta(\xi)]-\frac{c_{0}}{\sqrt{c_{0}^{2}-4}}} \\
& \times \exp \left\{-i\left[\frac{v \cos (2 v t)}{5-2 \sin (2 v t)} x^{2}+E \int_{0}^{t} d t^{\prime}\left(\frac{\gamma_{0}}{5-2 \sin \left(2 v t^{\prime}\right)}\right)^{2}\right]\right\}, \\
\Psi_{2}(x, t)= \pm & \sqrt{\frac{2 \sqrt{2} \gamma_{0} \lambda\left[E-1-q+q \operatorname{sn}^{2}(\xi, q)\right]}{[5-2 \sin (2 v t)] \sqrt{(E-1-q)(E-q)(E-1)}} \frac{\sqrt{c_{0}^{2}-4}}{\cosh [2 \lambda \Theta(\xi)]-c_{0}}} \\
& \times \exp \left\{-i\left[\frac{v \cos (2 v t)}{5-2 \sin (2 v t)} x^{2}+E \int_{0}^{t} d t^{\prime}\left(\frac{\gamma_{0}}{5-2 \sin \left(2 v t^{\prime}\right)}\right)^{2}\right]\right\},
\end{aligned}
$$

where $\Theta(\xi)=\sqrt{\frac{\left(E_{1}-q\right)\left(E_{1}-1\right)}{2 \lambda^{2}\left(E_{1}-1-q\right)}} \frac{\Pi\left(-\frac{q}{E_{1}-1-q} ; \operatorname{am}(\xi, q) \backslash q\right) \operatorname{dn}(\xi, q)}{\sqrt{1+q \operatorname{sn}^{2}(\xi, q)}}$, with $\Pi$ defined as the elliptic integral of the third kind and am $(\xi, \nu)$ the elliptic amplitude [28]. The profiles of the components are shown in Fig. 3. 
(a)

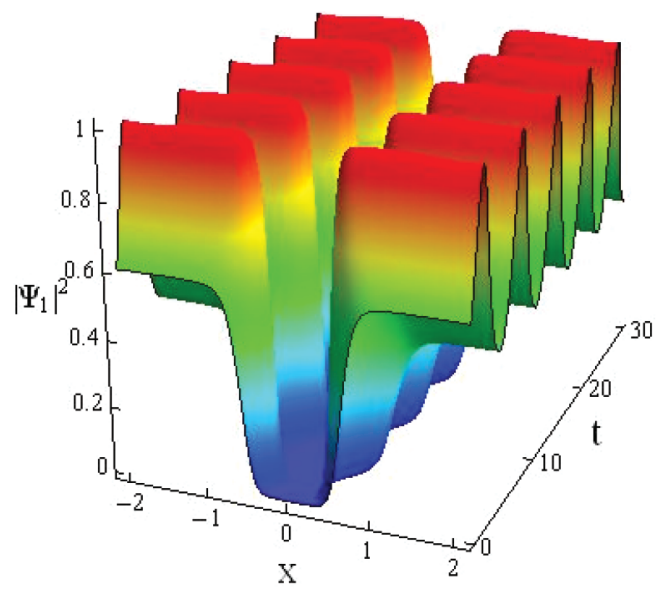

(b)

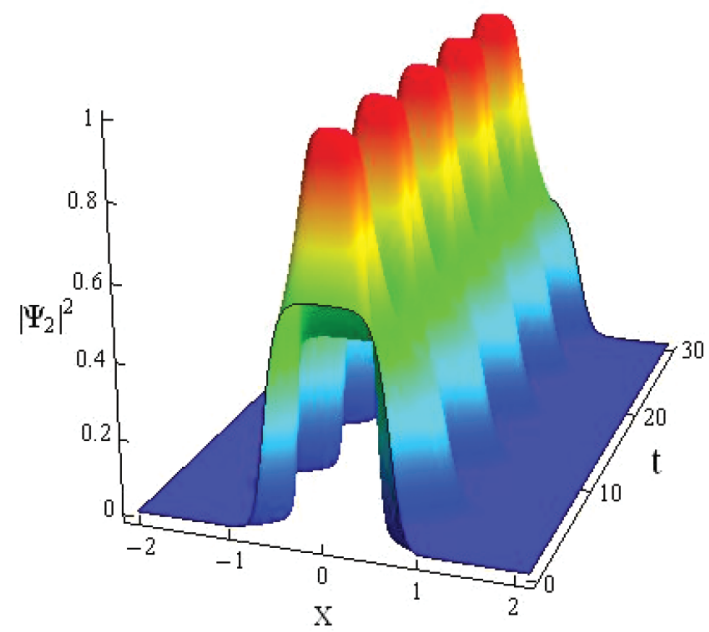

FIG. 3. (Color online) Components of the wide dark-bright vector soliton for BECs. (a) $\left|\Psi_{1}(x, t)\right|^{2}$, wide dark soliton from Eq. (33). (b) $\left|\Psi_{2}(x, t)\right|^{2}$, wide bright soliton from (34). With $\lambda=2.5, v=0.5, c_{0}=-2.0001, q=0.5, E_{1}=E_{2}=50$, and $\gamma_{0}=3$.

In the case B of vector solitons in a nonlinear optical fiber with Kerr nonlinearity only, the components of the wide dark-bright vector soliton are given by

$$
\begin{gathered}
\left|\Psi_{1}(x, t)\right|=\frac{\sqrt{\beta_{0}} \gamma_{0}\left(\Omega^{2}+\sigma^{2}\right) e^{\sigma t / 2}}{\left\{\sigma-e^{\sigma t}[\sigma \cos (\Omega t)+\Omega \sin (\Omega t)]+\beta_{0} \gamma_{0}\left(\Omega^{2}+\sigma^{2}\right)\right\}} \frac{\sinh (2 \lambda \xi)}{\cosh (2 \lambda \xi)-\frac{c_{0}}{\sqrt{c_{0}^{2}-4}}}, \\
\left|\Psi_{2}(x, t)\right|=\frac{\sqrt{\beta_{0}} \gamma_{0}\left(\Omega^{2}+\sigma^{2}\right) e^{\sigma t / 2}}{\left\{\sigma-e^{\sigma t}[\sigma \cos (\Omega t)+\Omega \sin (\Omega t)]+\beta_{0} \gamma_{0}\left(\Omega^{2}+\sigma^{2}\right)\right\}} \frac{2}{\sqrt{c_{0}^{2}-4} \cosh (2 \lambda \xi)-c_{0}},
\end{gathered}
$$

where $\xi=\frac{\beta_{0} \gamma_{0}^{2}\left(\Omega^{2}+\sigma^{2}\right)}{\sigma-e^{\sigma t}[\sigma \cos (\Omega t)+\Omega \sin (\Omega t)]+\beta_{0} \gamma_{0}\left(\Omega^{2}+\sigma^{2}\right)}$. Figure 4 shows the case when $\sigma \neq 0$; in this case the balance between the effects of the dispersion parameters, the nonlinearities, and the absorption or amplification coefficients is broken. In particular, we have chosen $\sigma<0$, which corresponds to an absorption that can be appreciated in the figure, which shows that the amplitude (depth) of $\left|\Psi_{2}\right|\left(\left|\Psi_{1}\right|\right)$ clearly diminishes with time.

(a)

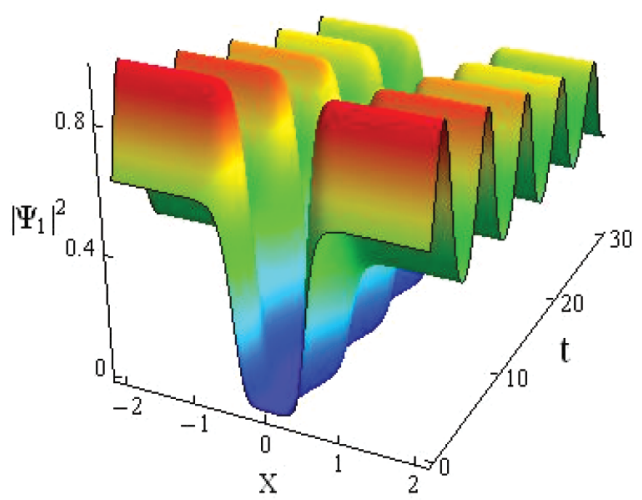

It is worthwhile to mention that the possibility of the pairing of bright and dark optical solitons under conditions of the XPM of waves in a nonlinear dispersing medium was shown analytically [30]. In that reference, it was predicted that under certain circumstances, novel quasiparticles can be produced as a result of nonlinear pairing of optical solitons. Different physical scenarios of the propagation and interaction of paired bright and dark solitons have been considered in [31-33]. The

(b)

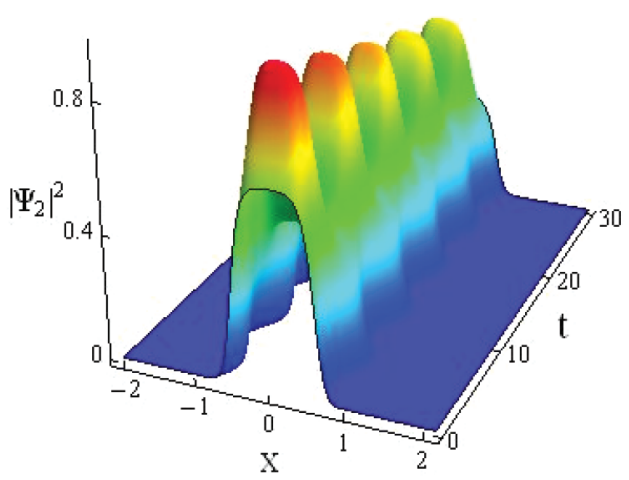

FIG. 4. (Color online) Components of a vector solution. (a) $\left|\Psi_{1}(x, t)\right|^{2}$ from (35), dark-type solution. (b) $\left|\Psi_{2}(x, t)\right|^{2}$ from (36), bright-type solution. With $\lambda=2, c_{0}=-2.0001, \gamma_{0}=3.1, \beta_{0}=1.6, \Omega=1$, and $\sigma=-0.01$. 

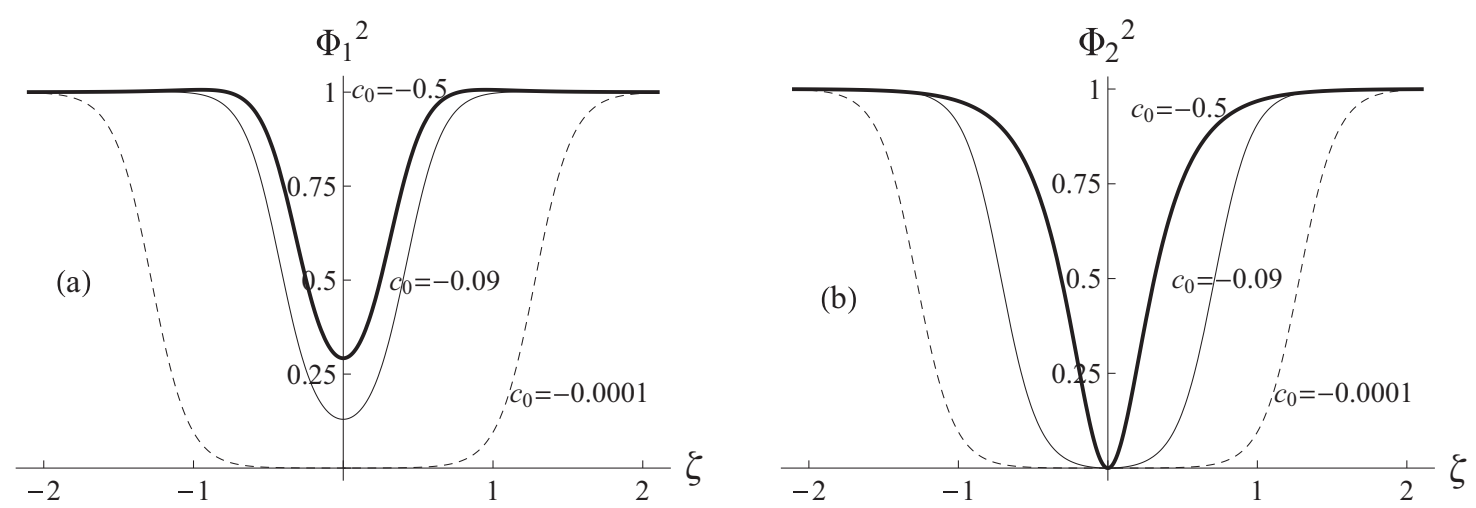

FIG. 5. (a) Gray-type soliton becomes a wide dark-type soliton. (b) Dark-type soliton becomes a wide dark-type soliton. With $\kappa=-1$ and $\rho=4$.

main physical mechanism of bright-dark soliton pairs (soliton molecules) was considered in detail in those pioneering papers. It is interesting to note that the soliton binding energy plays an important role in the soliton pairing, as has been shown recently in [34].

\section{Example 2: Wide vector solitons with cubic-quintic nonlinearities}

In this case, the system of differential equations (1) and (2) can model ultrashort pulse propagation along orthogonal polarization axes in a nonlinear optical fiber with both Kerr and non-Kerr nonlinearities. To find other solutions that solve Eqs. (19) by resorting to the orbit equation method, we choose $\mu_{1}=\mu_{2}=-\rho^{2}, G_{11}=4 \kappa \rho, G_{22}=4 \rho \lambda$, $G_{12}=G_{21}=6 \rho(\lambda+\kappa), H_{12}=2 F_{21}=6 \kappa(2 \lambda+3 \kappa), H_{21}=$ $2 F_{12}=6 \lambda(3 \lambda+2 \kappa), F_{11}=3 \kappa^{2}$, and $F_{22}=3 \lambda^{2}$. With this choice of parameters one can deal with (26), which now are the following first-order differential equations:

$$
\begin{aligned}
& \frac{d \Phi_{1}}{d \zeta}=\Phi_{2}\left(\lambda \Phi_{2}^{2}+3 \kappa \Phi_{1}^{2}+\rho\right), \\
& \frac{d \Phi_{2}}{d \zeta}=\Phi_{1}\left(3 \lambda \Phi_{2}^{2}+\kappa \Phi_{1}^{2}+\rho\right) .
\end{aligned}
$$

(a)

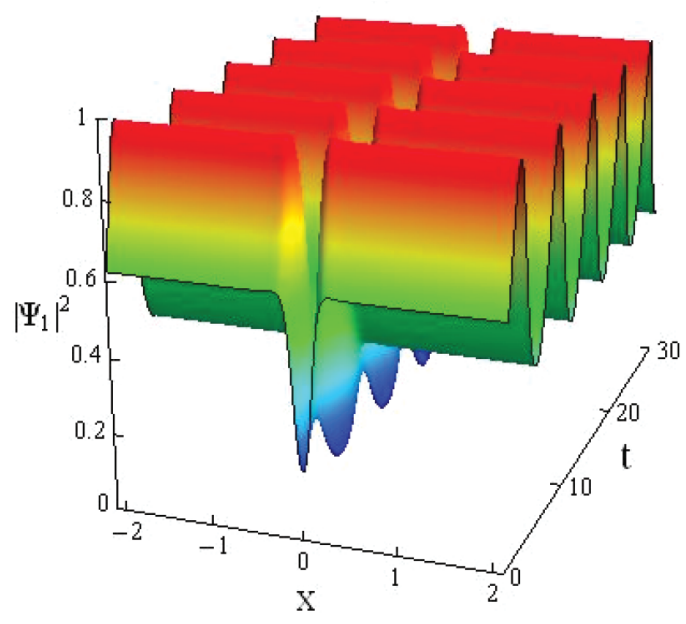

Then the orbit equation is

$$
\begin{aligned}
\left(\Phi_{2}^{2}\right. & \left.+\frac{\rho}{4 \lambda}\right)^{2 \sqrt{9 b^{2}-14 b+9}} \\
& \times\left(2 \varphi-\sqrt{9 b^{2}-14 b+9}\right)^{3(b+1)+\sqrt{9 b^{2}-14 b+9}} \\
& =c_{0}\left(2 \varphi+\sqrt{9 b^{2}-14 b+9}\right)^{3(b+1)-\sqrt{9 b^{2}-14 b+9}}
\end{aligned}
$$

where $c_{0}$ is a real arbitrary constant, $b=\frac{\lambda}{\kappa}$, and $\varphi=\frac{\Phi_{1}^{2}+\rho / 4 \kappa}{\Phi_{2}^{2}+\rho / 4 \lambda}-$ $\frac{3}{2}\left(\frac{\lambda}{\kappa}-1\right)$. This equation is reduced to a more manageable one when $\lambda=\kappa$. Then the orbit equation is of the form

$$
\Phi_{1}^{2}\left(\Phi_{1}^{2}-c_{0}\right)+\Phi_{2}^{2}\left(\Phi_{2}^{2}-c_{0}\right)-2 \Phi_{1}^{2} \Phi_{2}^{2}-c_{0} \frac{\rho}{2 \kappa}=0 .
$$

In this case, $c_{0}$ has two critical values, namely, 0 and $-\frac{2 \rho}{\kappa} \quad$ [20]. From (38) we find $\Phi_{1}^{2}=\frac{1}{2}\left[2 \Phi_{2}^{2}+c_{0}+\right.$ $\left.\sqrt{c_{0}\left(8 \Phi_{2}^{2}+c_{0}+\frac{2 \rho}{\kappa}\right)}\right]$. By substituting it in Eqs. (37) and integrating them we obtain $\Phi_{1}(\zeta)$ and $\Phi_{2}(\zeta)$, whose profiles are shown in Fig. 5. The profiles show how a gray-type soliton becomes a wide dark-type soliton and the dark-type soliton becomes a wide dark-type soliton when $c_{0}$ approaches its critical value 0 .

(b)

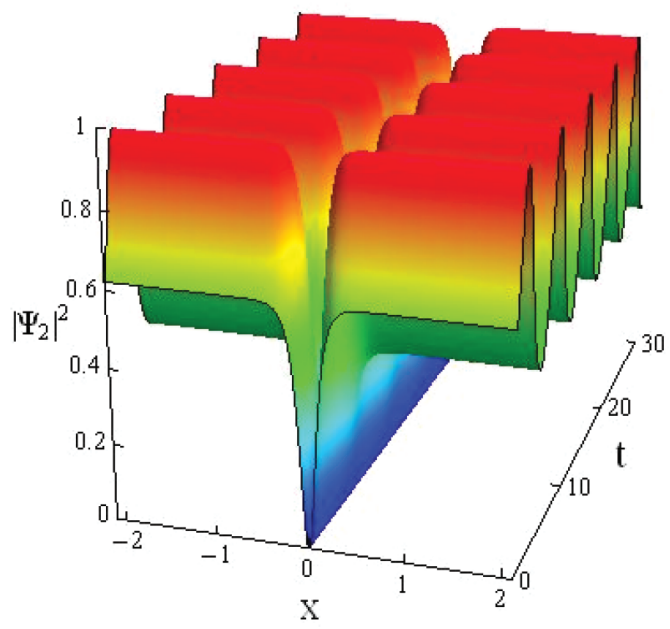

FIG. 6. (Color online) Components of the gray-dark vector soliton. (a) $\left|\Psi_{1}(x, t)\right|^{2}$, gray soliton. (b) $\left|\Psi_{2}(x, t)\right|^{2}$, dark soliton. With $\kappa=-1$, $\rho=4, \Omega=1, c_{0}=-0.5, \gamma_{0}=3.1, \beta_{0}=1.6$, and $\sigma=0$. 
(a)

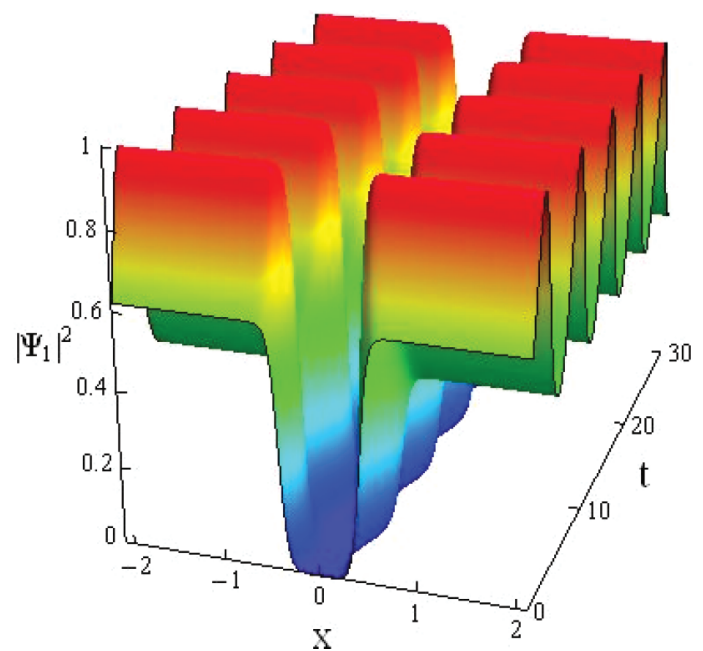

(b)

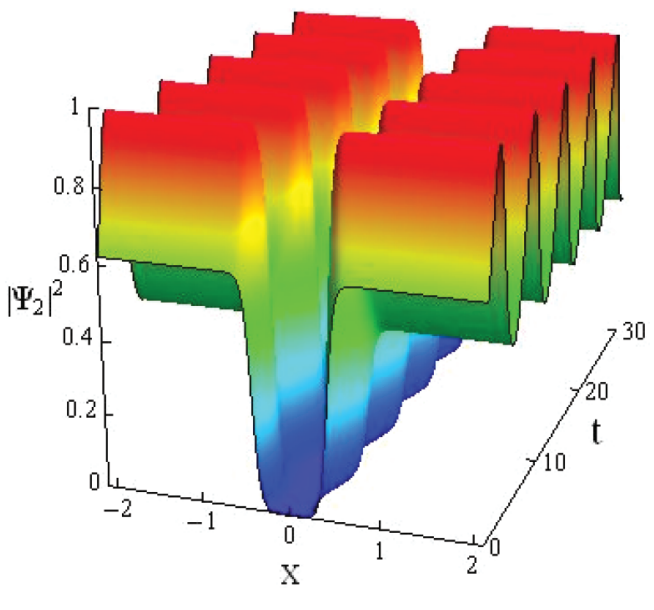

FIG. 7. (Color online) Components of the wide dark-dark vector soliton. (a) $\left|\Psi_{1}(x, t)\right|^{2}$, wide dark soliton. (b) $\left|\Psi_{2}(x, t)\right|^{2}$, wide dark soliton. With $\kappa=-1, \rho=4, \Omega=1, c_{0}=-0.0001, \gamma_{0}=3.1, \beta_{0}=1.6$, and $\sigma=0$.

By using the wave functions Eqs. (23) and by considering the case $\mathrm{B}$, we show the components of the gray-dark vector soliton in a nonlinear optical fiber in Fig. 6 for $\sigma=0$. The components of the wide dark-dark vector soliton in a nonlinear optical fiber are shown in Fig. 7 for $\sigma=0$. When $\sigma<0$ the components of the wide dark-dark vector soliton have the same behavior as in Fig. 4(a).

Now, we consider the second solution of Eq. (38), that is, $\Phi_{1}^{2}=\frac{1}{2}\left[2 \Phi_{2}^{2}+c_{0}-\sqrt{c_{0}\left(8 \Phi_{2}^{2}+c_{0}+\frac{2 \rho}{\kappa}\right)}\right]$. From Fig. 8 it is evident that a bright-type solution becomes a wide bright-type solution and a dark-type solution becomes a wide dark-type solution, when $c_{0}$ approaches its critical value $-\frac{2 \rho}{\kappa}$. The components of the wide dark-bright vector solution in a nonlinear optical fiber are presented in Fig. 9.

Detailed studies of the CQNLSE in both optics and BECs have been carried out in [35]. There, analytical soliton solutions are obtained and their stability and relation to dispersive shocks are analyzed. In those works the authors deal with autonomous models and report the appearance of coexisting dark and antidark (bright on a pedestal) solitons. Although we are treating nonautonomous systems here, we also have found such structures, namely, wide dark and antidark (wide bright on a pedestal), as shown in Figs. 8 and 9.

\section{Example 3: Periodic vector solitons with cubic-quintic nonlinearities}

Analytical periodic solutions can also be found. Those are solutions of the second-order differential equations only and, as such, we cannot use the orbit equation method, which relies upon first-order differential equations.

In particular, by choosing $\mu_{1}=\mu_{2}=-\sqrt{3} a^{2}, G_{11}=-1$, $G_{12}=G_{21}=b, G_{22}=-b^{2}$, and $F_{11}=F_{22}=-\frac{\sqrt{3}(2-\sqrt{3})}{\lambda^{4}} a^{2}$ where $b<0$, we have found

$$
\begin{aligned}
& \Phi_{1}(\zeta)=\frac{\sqrt{-b} \lambda}{\sqrt{1-\frac{3-\sqrt{3}}{2} \operatorname{sn}^{2}(\sqrt{2} a \zeta \mid 1 / 2)}}, \\
& \Phi_{2}(\zeta)=\frac{\lambda}{\sqrt{1-\frac{3-\sqrt{3}}{2} \operatorname{sn}^{2}(\sqrt{2} a \zeta \mid 1 / 2)}} .
\end{aligned}
$$

The amplitudes of these periodic solutions can also be modulated as was done in the previous examples.
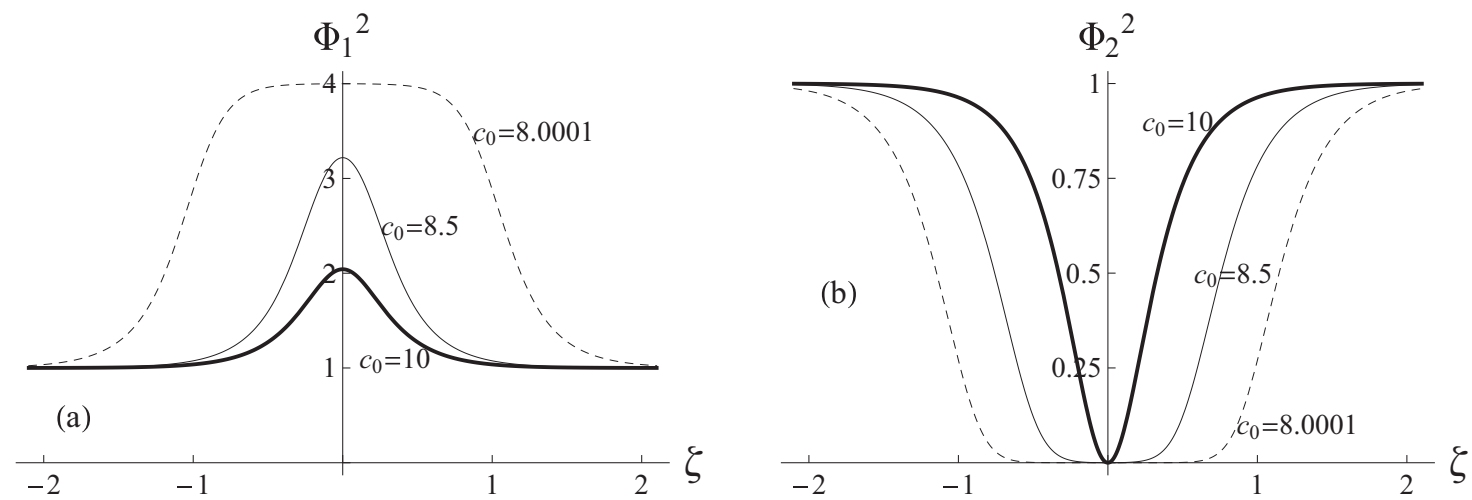

FIG. 8. (a) Bright-type solution becomes a wide bright-type solution. (b) Dark-type solution becomes a wide dark-type solution. With $\kappa=-1$ and $\rho=4$. 
(a)

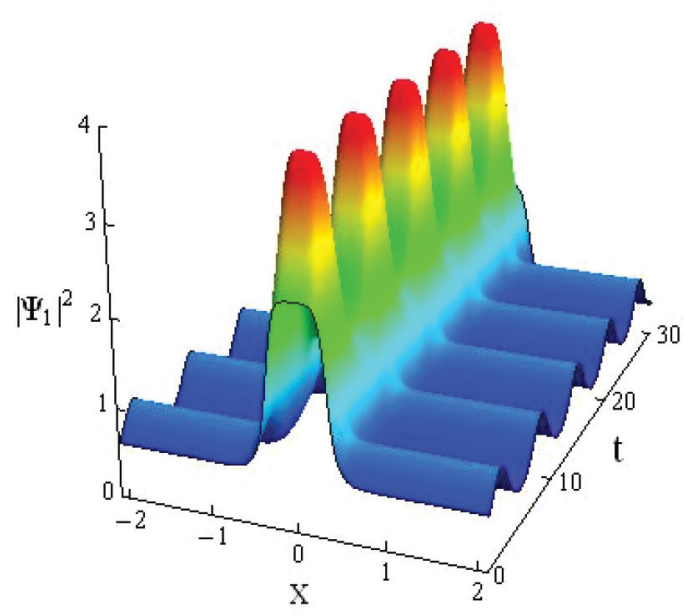

(b)

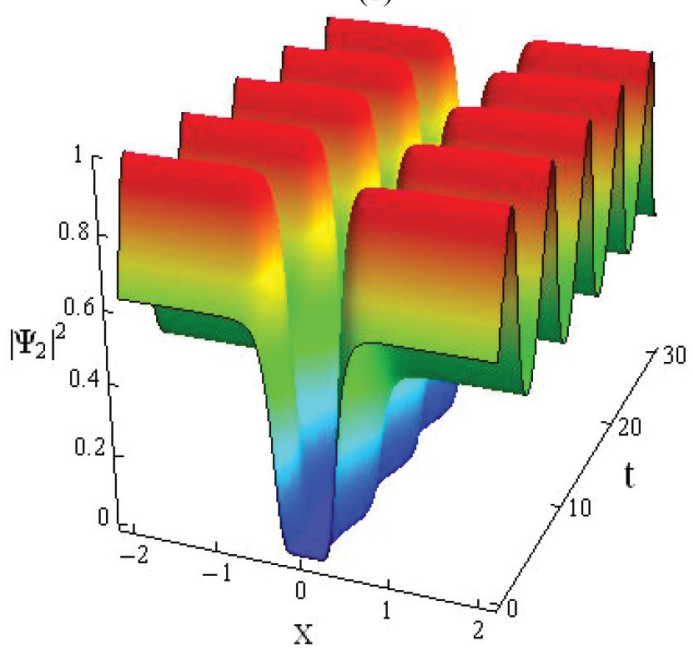

FIG. 9. (Color online) Components of the wide dark-bright vector soliton. (a) $\left|\Psi_{1}(x, t)\right|^{2}$, wide bright soliton. (b) $\left|\Psi_{2}(x, t)\right|^{2}$, wide dark soliton. With $\kappa=-1, \rho=4, c_{0}=8.0001, \gamma_{0}=3.1, \beta_{0}=1.6, \Omega=1$, and $\sigma=0$.

\section{CONCLUSIONS}

In this work we have extended the classes of exact solutions of two coupled nonautonomous nonlinear Schrödinger equations with cubic and quintic nonlinearities with time and space dependence. The solutions presented are of the following types: bright-dark, dark-dark, and gray-dark solitons and periodic-periodic solutions. In some of them the effects of absorption or amplification were also taken into account. Apart from the periodic solutions, one remarkable feature of the above configurations is the presence of an arbitrary integration constant $c_{0}$, which controls the width of the dark component and the width and amplitude of the bright component of the vector soliton. Physically acceptable solutions restrict the domain of validity of $c_{0}$, and for some values of $c_{0}$ (critical values) dark-dark vector solitons are found. Moreover, this constant is not a parameter of the model. It is related, instead, to the initial or boundary conditions of the vector soliton components and their first derivatives with respect to time and space. In this way, the width of the solitons is not dynamically controlled by $c_{0}$, but it can explain the possible appearance of wide vector solitons in physical systems whose dynamics is governed by coupled NLSEs.

Moreover, we would like to mention an interesting work dealing with multicomponent coupled solitons described by coherently coupled (autonomous) NLSEs [36]. In one specific case treated in that work, a bright-bright vector soliton solution, with one of the components exhibiting a flat-top profile very similar to $\Phi_{2}^{2}$ shown in Fig. 2, was found. Such kinds of bright flat-top solitons have also been reported in nonintegrable complex Ginzburg-Landau equations [37]. We think that a generalization of [36] to coherently coupled (nonautonomous) NLSEs may be possible and it will be considered in the near future.

Finally, in order to stimulate further experimental and theoretical studies in the field, it is important to discuss in detail the different physical effects involved in soliton coupling and pairing. For instance, one could consider the influence of disturbing factors such as linear absorption, high-order dispersion, the influence of the stimulated Raman self-scattering effect, and the modulation instability on the dynamics of bright and dark soliton pairing.

\section{ACKNOWLEDGMENTS}

The authors are indebted to the anonymous referees for excellent and constructive comments. L.E.A.M. thanks the Brazilian funding agency CAPES for support by a scholarship under the PEC-PG program. This work is also partially supported by CNPq (Grants No. 482043/2011-3, No. 304252/2011-5, and No. 306316/2012-9).
[1] G. P. Agrawal, Nonlinear Fiber Optics (Academic Press, San Diego, 2007).

[2] K. Bongs, S. Burger, S. Dettmer, D. Hellweg, J. Arlt, W. Ertmer, and K. Sengstock, Phys. Rev. A 63, 031602(R) (2001).

[3] V. E. Zakharov and A. B. Shabat, Zh. Eksp. Teor. Fiz. 61, 118 (1971) [Sov. Phys. JETP 34, 62 (1972)].

[4] W. J. Liu, B. Tia, T. Xu, K. Sun, and Y. Jiang, Ann. Phys. (NY) 325, 1633 (2010).
[5] V. N. Serkin and A. Hasegawa, Phys. Rev. Lett. 85, 4502 (2000); V. N. Serkin, T. L. Belyaeva, I. V. Alexandrov, and G. M. Melchor, Proc. SPIE 4271, 292 (2001).

[6] J. Belmonte-Beitia, V. M. Perez-Garcia, V. Vekslerchik, and V. V. Konotop, Phys. Rev. Lett. 100, 164102 (2008).

[7] A. T. Avelar, D. Bazeia, and W. B. Cardoso, Phys. Rev. E 79, 025602(R) (2009).

[8] L. E. Arroyo Meza, A. de Souza Dutra, and M. B. Hott, Phys. Rev. E 86, 026605 (2012). 
[9] N. J. Zabusky and M. D. Kruskal, Phys. Rev. Lett. 15, 240 (1965).

[10] V. N. Serkin, A. Hasegawa, and T. L. Belyaeva, Phys. Rev. Lett. 98, 074102 (2007); Phys. Rev. A 81, 023610 (2010); J. Mod. Opt. 57, 1456 (2010).

[11] J. L. Roberts, N. R. Claussen, James P. Burke, Jr., Chris H. Greene, E. A. Cornell, and C. E. Wieman, Phys. Rev. Lett. 81, 5109 (1998).

[12] M. Theis, G. Thalhammer, K. Winkler, M. Hellwig, G. Ruff, R. Grimm, and J. H. Denschlag, Phys. Rev. Lett. 93, 123001 (2004).

[13] V. Efimov, Phys. Lett. B 33, 563 (1970).

[14] D. Pushkarov and S. Tanev, Opt. Commun 124, 354 (1996).

[15] S. Burger, K. Bongs, S. Dettmer, W. Ertmer, K. Sengstock, A. Sanpera, G. V. Shlyapnikov, and M. Lewenstein, Phys. Rev. Lett. 83, 5198 (1999); J. Denschlag et al., Science 287, 97 (2000).

[16] D. J. Kaup and B. A. Malomed, Phys. Rev. A 48, 599 (1993); A. P. Sheppard and Y. S. Kivshar, Phys. Rev. E 55, 4773 (1997); Th. Busch and J. R. Anglin, Phys. Rev. Lett. 87, 010401 (2001); W. B. Cardoso, A. T. Avelar, D. Bazeia, and M. S. Hussein, Phys. Lett. A 374, 2356 (2010).

[17] W. B. Cardoso, A. T. Avelar, and D. Bazeia, Phys. Rev. E 86, 027601 (2012).

[18] D. Bazeia, M. J. dos Santos, and R. F. Ribeiro, Phys. Lett. A 208, 84 (1995); D. Bazeia, J. R. S. Nascimento, R. F. Ribeiro, and D. Toledo, J. Phys. A 30, 8157 (1997).

[19] A. de Souza Dutra, Phys. Lett. B 626, 249 (2005); A. de Souza Dutra and A. C. Amaro de Faria, Jr., ibid. 642, 274 (2006).

[20] L. E. Arroyo Meza, A. de Souza Dutra, J. R. L. dos Santos, M. B. Hott, and O. C. Winter, Europhys. Lett. 98, 10011 (2012).

[21] S. V. Manakov, Zh. Éksp. Teor. Fiz. 65, 505 (1973) [Sov. Phys. JETP 38, 248 (1974)].
[22] V. V. Afanasjev and V. N. Serkin, Sov. Lightwave Commun. 3, 101 (1993).

[23] V. V. Afanasjev and V. N. Serkin, Nonlinear Guided Wave Phenomena, 1993 OSA Technical Digest Series Vol. 15 (Optical Society of America, Washington, DC, 1993), paper TuB13.

[24] Y. Jiang, B. Tian, W. J. Liu, K. Sun, M. Li, and P. Wang, Phys. Rev. E 85, 036605 (2012).

[25] D. Bazeia and F. A. Brito, Phys. Rev. D 61, 105019 (2000).

[26] A. de Souza Dutra, A. C. Amaro de Faria, Jr., and M. Hott, Phys. Rev. D 78, 043526 (2008).

[27] F. S. Cataliotti et al., Science 293, 843 (2001); A. Trombettoni and A. Smerzi, Phys. Rev. Lett. 86, 2353 (2001); M. Greiner et al., Appl. Phys. B: Lasers Opt. 73, 769 (2001); Y. V. Kartashov, B. A. Malomed, and L. Torner, Rev. Mod. Phys. 83, 247 (2011).

[28] Handbook of Mathematical Functions, edited by M. Abramowitz and I. Stegun (Dover, Toronto, 1965).

[29] Ruiyu Hao et al., Opt. Commun. 245, 383 (2005).

[30] V. V. Afanas'ev, E. M. Dianov, A. M. Prokhorov, and V. N. Serkin, Pis'ma Zh. Eksp. Teor. Fiz. 48, 588 (1988) [JETP Lett. 48, 638 (1988)].

[31] S. Trillo, S. Wabnitz, E. M. Wright, and G. I. Stegeman, Opt. Lett. 13, 871 (1988).

[32] V. V. Afanasjev, E. M. Dianov, and V. N. Serkin, IEEE J. Quantum Electron. 25, 2656 (1989).

[33] V. V. Afanasjev, Y. S. Kivshar, V. V. Konotop, and V. N. Serkin, Opt. Lett. 14, 805 (1989).

[34] T. L. Belyaeva, V. N. Serkin C. Hernandez-Tenorio, and F. Garcia-Santibañez, J. Mod. Opt. 57, 1087 (2010).

[35] M. Crosta, A. Fratalocchi, and S. Trillo, Phys. Rev. A 84, 063809 (2011); M. Crosta, S. Trillo, and A. Fratalocchi, ibid. 85, 043607 (2012); Opt. Lett. 37, 1634 (2012).

[36] T. Kanna and K. Sakkaravarthi, J. Phys. A 44, 285211 (2011).

[37] N. Akhmediev and A. Ankiewicz, Solitons: Nonlinear Pulses and Beams (Chapman and Hall, London, 1997). 